\title{
The Classical Limit of Quantum Spin Systems
}

\author{
Elliott H. Lieb* \\ Institut des Hautes Etudes Scientifiques, Bures-sur-Yvette, France
}

Received February 28, 1973

\begin{abstract}
We derive a classical integral representation for the partition function, $Z^{Q}$, of a quantum spin system. With it we can obtain upper and lower bounds to the quantum free energy (or ground state energy) in terms of two classical free energies (or ground state energies). These bounds permit us to prove that when the spin angular momentum $J \rightarrow \infty$ (but after the thermodynamic limit) the quantum free energy (or ground state energy) is equal to the classical value. In normal cases. our inequality is $Z^{C}(J) \leqq Z^{Q}(J) \leqq Z^{C}(J+1)$.
\end{abstract}

\section{Introduction}

It is generally believed in statistical mechanics that if one takes a quantum spin system of $N$ spins, each having angular momentum $J$, normalizes the spin operators by dividing by $J$, and takes the limit $J \rightarrow \infty$, then one obtains the corresponding classical spin system wherein the spin variables are replaced by classical vectors and the trace is replaced by an integration over the unit sphere. Indeed, Millard and Leff [1] have shown this to be true for the Heisenberg model when $N$ is held fixed. Their proof is quite complicated and it is therefore not surprising that this goal was not achieved before 1971. Despite that success, however, the problem is not finished. One wants to show that one can interchange the limit $N \rightarrow \infty$ with the limit $J \rightarrow \infty$, i.e. is the classical system obtained if we first let $N \rightarrow \infty$ and then let $J \rightarrow \infty$ ? In the MillardLeff proof the control over the $N$ dependence of the error is not good enough to achieve this desideratum.

A more useful result, and one which would include the above, would be to obtain. for each $J$, upper and lower bounds to the quantum free energy in terms of the free energies of two classical systems such that those two bounds have a common classical limit as $J \rightarrow \infty$. In this paper we do just that, and the result is surprisingly simple: In most cases of interest (including the Heisenberg model), the classical upper bound is

* On leave from the Department of Mathematics, M.I.T., Cambridge, Mass. 02139, USA. Work partially supported by National Science Foundation Grant GP-31674X and by a Guggenheim Memorial Foundation Fellowship. 
obtained by replacing the quantum spin by $(J+1)$ times the classical unit vector, while the lower bound is obtained by using $J$ instead of $(J+1)$. Symbolically,

$$
Z^{C}(J) \leqq Z^{Q}(J) \leqq Z^{C}(J+1) .
$$

In other cases the result is a little more complicated to state, but it is of the same nature. With an upper and lower bound in hand, it is then possible to derive rigorous bounds on expectation values, as we shall describe in Sections V and VI.

The main tool in our derivation will be what has been termed by Arrechi et al. [2] the Bloch coherent state representation. These states and some of their properties were obtained earlier [3,4], but the most complete account is in Ref. [2]. Our lower bound is obtained by a variational calculation, while the upper bound is obtained from a representation of the quantum partition function that bears some similarity to the Wiener (or path) integral. Apart from its use in deriving the upper bound, the representation may be of theoretical value in proving other properties of quantum spin systems. In particular, it provides a sensible definition of the quantum partition function for all complex $J$, not just when $J$ is half an integer, and one may discuss the existence or non-existence of a phase transition as a function of the continuous parameter $J$.

In a forthcoming paper [7] it will be shown how to apply the methods and bounds developed herein (using not only the Bloch states but the Glauber coherent photon states as well) to certain models of the interaction of atoms with a quantized radiation field, for example the Dicke Maser model.

\section{Bloch Coherent States}

In this section we recapitulate results derived in Refs. [2] and [3]. We consider a single quantum spin of fixed total angular-momentum and shall denote by $S \equiv\left(S_{x}, S_{y}, S_{z}\right)$ the usual angular momentum operators:

$$
\left[S_{x}, S_{y}\right]=i S_{z}, \quad \text { and cyclically, } S_{ \pm}=S_{x} \pm i S_{y} .
$$

We denote by $J$ the total angular momentum, i.e.

$$
S^{2}=S_{x}{ }^{2}+S_{y}{ }^{2}+S_{z}{ }^{2}=J(J+1) .
$$

The Hilbert space on which these operators act has dimension $2 J+1$, i.e. it is $\mathbb{C}^{2 J+1}$. 
On the classical side, we denote by $\mathscr{S}$ the unit sphere in three dimensions:

$$
\mathscr{S}=\left\{(x, y, z) \mid x^{2}+y^{2}+z^{2}=1\right\},
$$

and by $L^{2}(\mathscr{S})$ the space of square integrable functions on $\mathscr{S}$ with the usual measure

$$
\begin{aligned}
\Omega & =(\theta, \varphi), 0 \leqq \theta \leqq \pi, 0 \leqq \varphi<2 \pi, \\
d \Omega & =\sin \theta d \theta d \varphi, \\
x & =\sin \theta \cos \varphi, y=\sin \theta \sin \varphi, z=\cos \theta .
\end{aligned}
$$

(Note: In Ref. [2], but not Ref. [3] the "south pole", instead of the customary "north pole" corresponds to $\theta=0$. Hence our formulas will differ from Ref. [2] by the replacement $\theta \rightarrow \pi-\theta$ ).

With $|J\rangle \in \mathbb{C}^{2 J+1}$ being a normalized "spin up" state, $S_{z}|J\rangle=J|J\rangle$. one defines the Bloch state $|\Omega\rangle \in \mathbb{C}^{2 J+1}$ by

$$
\begin{aligned}
|\Omega\rangle & =\exp \left\{\frac{1}{2} \theta\left[S_{-} e^{i \varphi}-S_{+} e^{-i \varphi}\right]\right\}|J\rangle \\
& =\left[\cos \frac{1}{2} \theta\right]^{2 J} \exp \left\{\left(\tan \frac{1}{2} \theta\right) e^{i \varphi} S_{-}\right\}|J\rangle \\
& =\sum_{M=-J}^{J}\left(\begin{array}{c}
2 J \\
M+J
\end{array}\right)^{1 / 2}\left(\cos \frac{1}{2} \theta\right)^{J+M}\left(\sin \frac{1}{2} \theta\right)^{J-M} \exp [i(J-M) \varphi]|M\rangle
\end{aligned}
$$

where $|M\rangle$ is the normalized state

$$
|M\rangle=\left(\begin{array}{c}
2 J \\
M+J
\end{array}\right)^{-1 / 2}[(J-M) !]^{-1}\left(S_{-}\right)^{J-M}|J\rangle
$$

such that

$$
S_{z}|M\rangle=M|M\rangle .
$$

It is clear from (2.7) that the set of states $|\Omega\rangle$ are complete in $\mathbb{C}^{2 J+1}$. Their overlap is given by

$$
\begin{aligned}
K_{J}\left(\Omega^{\prime}, \Omega\right) & \equiv\left\langle\Omega^{\prime} \mid \Omega\right\rangle \\
& =\left\{\cos \frac{1}{2} \theta \cos \frac{1}{2} \theta^{\prime}+e^{i\left(\varphi-\varphi^{\prime}\right)} \sin \frac{1}{2} \theta \sin \frac{1}{2} \theta^{\prime}\right\}^{2 J}
\end{aligned}
$$

so that if we think of $K_{J}\left(\Omega^{\prime}, \Omega\right)$ as the kernel of a linear transformation on $L^{2}(\mathscr{S})$ it is selfadjoint and compact. In fact, it is positive semidefinite. We also have

where

$$
\left|K_{J}\left(\Omega^{\prime}, \Omega\right)\right|^{2}=\left[\cos \frac{1}{2} \Theta\right]^{4 J},
$$

$$
\cos \Theta=\cos \theta \cos \theta^{\prime}+\sin \theta \sin \theta^{\prime} \cos \left(\varphi-\varphi^{\prime}\right)
$$


is the cosine of the angle between $\Omega$ and $\Omega^{\prime}$. In particular $|\Omega\rangle$ is normalized since $K_{J}(\Omega, \Omega)=1$.

Now let $\mathscr{M}^{2 J+1}$ be the set of linear transformations on $\mathbb{C}^{2 J+1}$ (i.e. operators on the spin space) and, for a given $G \in L^{1}(\mathscr{P})$, define $A_{G} \in \mathscr{M}^{2 J+1}$ by

$$
A_{G}=\frac{2 J+1}{4 \pi} \int d \Omega G(\Omega)|\Omega\rangle\langle\Omega| .
$$

(Note: $\int d \Omega$ always means $\int_{\mathscr{S}} d \Omega$ ). Since the Hilbert space is finite dimensional there is no problem in giving a meaning to (2.13). It is a remarkable fact that every operator in $\mathscr{M}^{2 J+1}$ can be written in the form (2.13). In particular,

$$
\mathbf{1}=\frac{2 J+1}{4 \pi} \int d \Omega|\Omega\rangle\langle\Omega| .
$$

Thus, to every operator $A \in \mathscr{M}^{2 J+1}$ there correspond two functions:

$$
g(\Omega)=\langle\Omega|A| \Omega\rangle,
$$

and the $G(\Omega)$ of (2.13). The former is, of course, unique, but the latter is not. However, it is always possible to choose $G(\Omega)$ to be infinitely differentiable. In Table 1 we list some function pairs for operators of common interest and useful formulas for calculation are given in Appendix A.

Table 1. Expectation values, $y(\Omega)$, and operator kernels, $G(\Omega)$. [cf. (2.13), (2.15)] for various operators commonly appearing in quantum spin Hamiltonians

\begin{tabular}{lll}
\hline Operator & $g(\Omega),(2.15)$ & $G(\Omega),(2.13)$ \\
\hline$S_{z}$ & $J \cos \theta$ & $(J+1) \cos \theta$ \\
$S_{\mathrm{x}}$ & $J \sin \theta \cos \varphi$ & $(J+1) \sin \theta \cos \varphi$ \\
$S_{y}$ & $J \sin \theta \sin \varphi$ & $(J+1) \sin \theta \sin \varphi$ \\
$S_{z}{ }^{2}$ & $J\left(J-\frac{1}{2}\right)(\cos \theta)^{2}+J / 2$ & $(J+1)(J+3 / 2)(\cos \theta)^{2}-\frac{1}{2}(J+1)$ \\
$S_{x}{ }^{2}$ & $J\left(J-\frac{1}{2}\right)(\sin \theta \cos \varphi)^{2}+J / 2$ & $(J+1)(J+3 / 2)(\sin \theta \cos \varphi)^{2}-\frac{1}{2}(J+1)$ \\
$S_{y}{ }^{2}$ & $J\left(J-\frac{1}{2}\right)(\sin \theta \cos \varphi)^{2}+J / 2$ & $(J+1)(J+3 / 2)(\sin \theta \cos \varphi)^{2}-\frac{1}{2}(J+1)$ \\
\hline
\end{tabular}

We need three final remarks. The first is that if we consider $|\Omega\rangle\left\langle\Omega^{\prime}\right| \in \mathscr{M}^{2 J+1}$ then

$$
\operatorname{Tr}|\Omega\rangle\left\langle\Omega^{\prime}\right|=K_{J}\left(\Omega^{\prime}, \Omega\right)
$$


(where Tr means Trace) as may be seen from (2.7). Hence, from (2.13)

$$
\operatorname{Tr} A_{G}=\frac{2 J+1}{4 \pi} \int d \Omega G(\Omega) .
$$

The second is that

$$
\frac{2 J+1}{4 \pi} \int d \Omega K_{J}\left(\Omega^{\prime}, \Omega\right) K_{J}\left(\Omega, \Omega^{\prime \prime}\right)=K_{J}\left(\Omega^{\prime}, \Omega^{\prime \prime}\right),
$$

as may be seen from (2.14). Thus, $K_{J}$ reproduces itself under convolution.

The third remark is that for any $A \in \mathscr{M}^{2 J+1}$ we can use (2.14) to obtain

$$
\begin{aligned}
\operatorname{Tr} A & =\frac{2 J+1}{4 \pi} \int d \Omega \operatorname{Tr}|\Omega\rangle\langle\Omega| A \\
& =\frac{2 J+1}{4 \pi} \int d \Omega \sum_{M=-J}^{J}\langle M \mid \Omega\rangle\langle\Omega|A| M\rangle \\
& =\frac{2 J+1}{4 \pi} \int d \Omega\langle\Omega|A| \Omega\rangle .
\end{aligned}
$$

\section{Lower Bound to the Quantum Partition Function}

We consider a system of $N$ quantum spins and shall label the operators and the angular momenta (which need not all be the same) by a superscript $i, i=1, \ldots, N$. The Hamiltonian, $H$, can be completely general but, in any event, it can always be written as a polynomial in the $3 N$ spin operators. The partition function is

where

$$
Z^{Q}=\alpha_{N} \operatorname{Tr} \exp (-\beta H),
$$

$$
\alpha_{N}=\prod_{i=1}^{N}\left(2 J^{i}+1\right)^{-1} \text {. }
$$

[The normalization factor $\alpha_{N}$ is inessential; it is chosen to agree with the classical partition function when $\beta=0]$. The Hilbert space is

$$
\mathscr{H}_{N}=\bigotimes_{i=1}^{N} \mathscr{H}^{i}=\bigotimes_{i=1}^{N} \mathbb{C}^{2 J^{i+1}} \text {. }
$$

We denote by $\left|\Omega_{N}\right\rangle$ the complete, normalized set of states on $\mathscr{H}_{N}$ defined by

$$
\left|\Omega_{N}\right\rangle=\bigotimes_{i=1}^{N}\left|\Omega^{i}\right\rangle
$$


by $\mathscr{S}_{N}$ the Cartesian product of $N$ copies of the unit sphere, and by $d \Omega_{N}$ the product measure (2.4), (2.5) and (2.6) on $\mathscr{S}_{N}$. Using (2.19),

$$
Z^{Q}=(4 \pi)^{-N} \int d \Omega_{N}\left\langle\Omega_{N}\left|e^{-\beta H}\right| \Omega_{N}\right\rangle .
$$

By the Peierls-Bogoliubov inequality, $\left\langle\psi\left|e^{X}\right| \psi\right\rangle \geqq \exp \langle\psi|X| \psi\rangle$ for any normalized $\psi \in \mathscr{H}_{N}$ and $X$ selfadjoint. Thus,

$$
Z^{Q} \geqq(4 \pi)^{-N} \int d \Omega_{N} \exp \left\{-\beta\left\langle\Omega_{N}|H| \Omega_{N}\right\rangle\right\} .
$$

Suppose, at first, that the polynomial, $H$, is linear in the operators $S^{i}$ of each spin. That is we allow multiple site interactions of arbitrary complexity such as $S_{x}{ }^{1} S_{y}{ }^{2} S_{y}{ }^{3} S_{z}{ }^{4}$, but do not allow monomials such as $\left(S_{x}{ }^{1}\right)^{2}$ or $S_{x}{ }^{1} S_{y}{ }^{1}$. In this case, which we shall refer to as the normal case, we see from (2.15) and Table 1 that the right side of (3.6) is precisely the classical partition function in which each $S^{i}$ is replaced by $J^{i}$ times a vector in $\mathscr{S}$. I.e.

$$
\boldsymbol{S}^{i} \rightarrow J^{i}\left(\sin \theta^{i} \cos \varphi^{i}, \sin \theta^{i} \sin \varphi^{i}, \cos \theta^{i}\right) .
$$

Thus, in the normal case,

$$
Z^{Q} \geqq Z^{C}\left(J^{1}, \ldots, J^{N}\right),
$$

where $Z^{C}$ means the classical partition function (with the normalization $\left.(4 \pi)^{-N}\right)$.

In more complicated cases, (3.7) is not correct and $S_{z}{ }^{1}$, for example, has to be replaced by $J^{1} \cos \theta^{1}$ if it appears linearly in $H,\left(S_{z}{ }^{1}\right)^{2}$ has to be replaced by $\left[J^{1} \cos \theta^{1}\right]^{2}+J^{1}\left(\sin \theta^{1}\right)^{2} / 2$ and so forth (see Table 1$)$. However, to leading order in $J^{i}$, (3.7) is correct.

We note in passing that it is not necessary to use the Peierls-Bogoliubov inequality for all operators appearing in $H$. Thus, suppose the whole Hilbert space is $\mathscr{H}^{\prime}=\mathscr{H} \otimes \mathscr{H}_{N}$ where $\mathscr{H}$ is the Hilbert space of some additional degrees of freedom (which may or may not themselves be spins) and $H$ is selfadjoint on $\mathscr{H}^{\prime}$. Then (by a generalized PeierlsBogoliubov inequality)

$$
\begin{aligned}
Z^{Q} & =\alpha_{N} \operatorname{Tr}_{\mathscr{H}} \operatorname{Tr}_{\mathscr{H}} \exp (-\beta H) \\
& \geqq \operatorname{Tr}_{\mathscr{H}}(4 \pi)^{-N} \int d \Omega_{N} \exp \left\{-\beta\left\langle\Omega_{N}|H| \Omega_{N}\right\rangle\right\}
\end{aligned}
$$

where $\left\langle\Omega_{N}|H| \Omega_{N}\right\rangle$ is a partial expectation value and defines a selfadjoint operator on $\mathscr{H}$. We shall give an example of (3.9) in Appendix B. It is clear that if $\mathscr{H}$ is itself a spin space, then (3.9) gives a better bound than (3.6) applied to the full space $\mathscr{H}^{\prime}$. 


\section{Upper Bound to the Quantum Partition Function}

Returning to the definitions (3.1) and (3.3) we note that

where

$$
Z^{Q}=\lim _{n \rightarrow \infty} Z(n),
$$

$$
Z(n)=\alpha_{N} \operatorname{Tr}\left(\mathbf{1}-\beta n^{-1} H\right)^{n} .
$$

Now, let $H$ be represented by some $G\left(\Omega_{N}\right)$ as in (2.13), whence $\mathbf{1}-\beta n^{-1} H$ is represented by

$$
F_{n}\left(\Omega_{N}\right)=1-\beta n^{-1} G\left(\Omega_{N}\right) .
$$

Using (2.10), (2.13) and (2.16), we can represent $Z_{n}$ as an $n N$ fold integral:

$$
Z(n)=\alpha_{N} \int d \Omega_{N}{ }^{1} \cdots \int d \Omega_{N}{ }^{n} \prod_{j=1}^{n} F_{n}\left(\Omega_{N}{ }^{j}\right) L_{J}\left(\Omega_{N}{ }^{j}, \Omega_{N}{ }^{j+1}\right)
$$

with $n+1 \equiv 1$ in the last factor, and where

$$
L_{J}\left(\Omega_{N}{ }^{\prime}, \Omega_{N}\right) \equiv(4 \pi)^{-N} \alpha_{N}{ }^{-1} \prod_{i=1}^{N} K_{J^{i}}\left(\Omega^{\prime i}, \Omega^{i}\right) .
$$

Thus

$$
\begin{gathered}
L_{J}\left(\Omega_{N}, \Omega_{N}\right)=(4 \pi)^{-N} a_{N}{ }^{-1} . \\
\int d \Omega_{N} L_{J}\left(\Omega_{N}{ }^{\prime}, \Omega_{N}\right) L_{J}\left(\Omega_{N}, \Omega_{N}{ }^{\prime \prime}\right)=L_{J}\left(\Omega_{N}{ }^{\prime}, \Omega_{N}{ }^{\prime \prime}\right) .
\end{gathered}
$$

Equations (4.1) and (4.4) are our desired integral representation for $Z^{Q}$. To use them to obtain a bound, we think of $F_{n}$ as a multiplication operator and of $L_{J}$ as the kernel of a compact, selfadjoint operator on $L^{2}\left(\mathscr{S}_{N}\right)$. If $B\left(\Omega_{N}{ }^{\prime}, \Omega_{N}\right)$ is such a kernel, then

$$
\operatorname{Tr} B=\int d \Omega_{N} B\left(\Omega_{N}, \Omega_{N}\right)
$$

is the trace on $L^{2}\left(\mathscr{S}_{N}\right)$. Thus,

$$
Z(n)=\alpha_{N} \operatorname{Tr}\left(F_{n} L_{J}\right)^{n} .
$$

In general, if $m=2^{j}, j=0,1,2,3, \ldots$,

$$
\left|\operatorname{Tr}(A B)^{2 m}\right| \leqq \operatorname{Tr}\left(A^{2} B^{2}\right)^{m} \leqq \operatorname{Tr} A^{2 m} B^{2 m},
$$

whenever $A$ and $B$ are selfadjoint. This follows from the Schwarz inequality (see Ref. [5] for details). Hence, if we take a sequence $n=2^{j}$, $j=1,2, \ldots$ in (4.2) and use (4.7) $n$ times and (4.6), we obtain, in the limit 
$n \rightarrow \infty$,

$$
Z^{Q} \leqq(4 \pi)^{-N} \int d \Omega_{N} \exp \left[-\beta G\left(\Omega_{N}\right)\right]
$$

(4.11) is our desired classical upper bound. It is just like (3.6). In the normal case we see from Table 1 that $\boldsymbol{S}^{i}$ is replaced by $\left(J^{i}+1\right)$ times a classical unit vector. In other cases, $G\left(\Omega_{N}\right)$ is a bit more complicated, but the same remarks as in Section III apply. Thus, in the normal case

$$
Z^{C}\left(J^{1}, \ldots, J^{N}\right) \leqq Z^{Q} \leqq Z^{C}\left(J^{1}+1, \ldots, J^{N}+1\right)
$$

This inequality says that as $J$ increases the quantum and classical free energies form two decreasing, interlacing sequences.

As in Section III, if $\mathscr{H}^{\prime}=\mathscr{H} \otimes \mathscr{H}_{N}$ an inequality similar to (4.11) can be shown to hold, i.e.

$$
Z^{Q} \leqq \operatorname{Tr}_{\mathscr{H}}(4 \pi)^{-N} \int d \Omega_{N} \exp \left[-\beta H\left(\cdot, \Omega_{N}\right)\right]
$$

where $H\left(\cdot, \Omega_{N}\right)$ is a selfadjoint operator on $\mathscr{H}$ obtained by replacing each monomial in the spin operators in $H$ by the appropriate $G\left(\Omega_{N}\right)$ function found in Table 1. We shall illustrate (4.13) in Appendix B. If $\mathscr{H}$ is a spin space then (4.13) gives a better bound than (4.11) applied to the full $\mathscr{H}^{\prime}$.

\section{Bounds on Expectation Values and the Ground State Energy}

The expectation value of a quantum operator (observable), $A$, is

$$
\langle A\rangle^{Q}=\operatorname{Tr} A \exp (-\beta H) / \operatorname{Tr} \exp (-\beta H) .
$$

We can always assume $A$ is selfadjoint (otherwise consider $A+A^{\dagger}$ and $i A-i A^{\dagger}$ ), in which case the Peierls-Bogoliubov inequality reads, for $\lambda$ real,

$$
\lambda\langle A\rangle^{Q} \geqq f(\lambda)-f(0),
$$

where

$$
f(\lambda)=-\beta^{-1} \ln \operatorname{Tr} \exp [-\beta(H+\lambda A)],
$$

is a free energy. Hence, with $\lambda>0$,

$$
[f(0)-f(-\lambda)] / \lambda \geqq\langle A\rangle^{Q} \geqq[f(\lambda)-f(0)] / \lambda .
$$

The upper and lower bounds to $f(\lambda)$ derived in the preceding two sections can be used to advantage in (5.4). In particular, we use (5.4) in the next section to derive $J \rightarrow \infty$ limits of quantum expectation values. 
If we take the limit $\beta \rightarrow \infty$ in (3.1) we obtain bounds on the quantum ground state energy:

$$
E_{-}^{C} \leqq E^{Q} \leqq E_{+}^{C},
$$

where $E^{C}$ is the classical ground state energy (i.e. the minimum of the classical Hamiltonian over $\mathscr{S}_{N}$ ) and the + (resp. -) refers to the substitution of the appropriate $G\left(\Omega_{N}\right)$ (resp. $g\left(\Omega_{N}\right)$ ) functions from Table 1. In the normal case

$$
E^{C}\left(J^{1}, \ldots, J^{N}\right) \geqq E^{Q} \geqq E^{C}\left(J^{1}+1, \ldots, J^{N}+1\right) .
$$

As ground state expectation values obey an inequality similar to (5.2), with $f$ replaced by $E$, a bound similar to (5.4) holds for $E$. This is merely the variational principle.

The upper bound in (5.6) is easy to obtain directly by a variational calculation, but the lower bound is not. It is not easy to find a direct proof of it in a system consisting of three spins antiferromagnetically coupled to each other.

\section{The Thermodynamic Limit}

A. The Free Energy

We shall, for simplicity, consider only the normal case here. The general case can be handled in a similar manner.

Let $H_{N}$ be a Hamiltonian (polynomial) of $N$ spins in which each spin has angular momentum one. Replace each spin operator $S^{i}$ by $(J)^{-1} S^{i}$ and let $S^{i}$ now have angular momentum $J$. We shall denote this symbolically by $H_{N}^{Q}(J)$ and the partition function, (3.1), by $Z_{N}^{Q}(J)$. [It would equally be possible to allow different $J$ values for different spins, but that is a needless complication. Also, the factor $J^{-1}$ is not crucial. One could as well use $\left.J^{-1 / 2}(J+1)^{-1 / 2}\right]$. Denoting the free energy per spin by $f_{N}(J)=-(N \beta)^{-1} \ln Z_{N}(J)$, the theorem to be proved is that

$$
\lim _{J \rightarrow \infty} \lim _{N \rightarrow \infty} f_{N}^{Q}(J)=f^{C} \equiv \lim _{N \rightarrow \infty} f_{N}^{C},
$$

where $f_{N}^{C}$ is the free energy per spin of the classical partition function in which each $S^{i}$ is replaced by a classical unit vector. It is assumed that $H_{N}$ is known to have a thermodynamic limit for the free energy per spin. We also want to prove an analogous formula for the ground state energy per spin. Our bounds are

$$
f_{N}^{C} \geqq f_{N}^{C}(J) \geqq f_{N}^{C}\left(\delta_{J}\right),
$$


where the right side is the classical free energy per spin in which each vector is multiplied by $\delta_{J} \equiv(J+1) / J$.

If we think of $\delta_{J}$ as a variable, $\delta$, then $H_{N}^{C}(\delta)$, the classical Hamiltonian as a function of $\delta$. is continuous in $\delta$. Moreover, $N^{-1} H_{N}^{C}(\delta)$ is equicontinuous in $N$, i.e. given any $\varepsilon>0$ it is possible to find a $\gamma>0$ such that $\left\|N^{-1}\left[H_{N}^{C}(\delta+x)-H_{N}^{C}(\delta)\right]\right\| \leqq \varepsilon$ for $|x|<\gamma$, independent of $N$, where \|\| means the uniform on $\mathscr{S}_{N}$. Hence, the limit function

$$
f^{C}(\delta) \equiv \lim _{N \rightarrow \infty} f_{N}^{C}(\delta)
$$

is continuous in $\delta$. This, together with (6.2), proves (6.1).

The same equicontinuity holds for the classical ground state energy. Thus, the analogue of (6.1) is also true for the ground state energy per spin:

$$
\lim _{J \rightarrow \infty} \lim _{N \rightarrow \infty} N^{-1} E_{N}^{Q}(J)=\lim _{N \rightarrow \infty} E_{N}^{C} .
$$

\section{B. Expectation Values}

We consider expectation values of intensive observables $N^{-1} A_{N}$. For example, $A_{N}$ might be the Hamiltonian itself, in which case $\left\langle N^{-1} A_{N}\right\rangle$ is the energy per spin. Alternatively, $A_{N}$ could be $\sum_{i=1}^{N} S_{z}^{i}$ so that $\left\langle N^{-1} A_{N}\right\rangle$ is the magnetization per spin. As before, we replace each $S^{i}$ by $(J)^{-1}$ times a quantum spin of angular momentum $J$, both in the Hamiltonian and in $A_{N}$. Then, using inequality (5.4) and the bounds (6.2) we have, for each positive $\lambda$, fixed $N$ and fixed $J$,

$$
\begin{aligned}
\lambda^{-1}\left[f_{N}^{C}(0 ; 1)-f_{N}^{C}\left(-\lambda ; \delta_{J}\right)\right] & \geqq N^{-1}\left\langle A_{N}\right\rangle^{Q} \\
& \geqq \lambda^{-1}\left[f_{N}^{C}\left(\lambda ; \delta_{J}\right)-f_{N}^{C}(0 ; 1)\right],
\end{aligned}
$$

where $f_{N}^{C}(\lambda ; \delta)$ is the classical free energy per spin when the Hamiltonian is $H_{N}^{C}+\lambda A_{N}^{C}$ and where each classical spin unit vector in $H_{N}^{C}$ and $A_{N}^{C}$ is multiplied by $\delta$. We are interested in $\delta_{J}=(J+1) / J$.

Now take the limit $N \rightarrow \infty$ and then the limit $J \rightarrow \infty$ in (6.5). By the same equicontinuity remark as in Section VI.A, for each $\lambda>0$,

$$
\begin{gathered}
\limsup _{J \rightarrow \infty} \limsup _{N \rightarrow \infty} N^{-1}\left\langle A_{N}\right\rangle^{Q} \leqq \lambda^{-1}\left[f^{C}(0)-f^{C}(-\lambda)\right] . \\
\liminf _{J \rightarrow \infty} \liminf _{N \rightarrow \infty} N^{-1}\left\langle A_{N}\right\rangle^{Q} \geqq \lambda^{-1}\left[f^{C}(\lambda)-f^{C}(0)\right] .
\end{gathered}
$$

In (6.5), $f^{C}(\lambda)$ is the limiting classical free energy per spin for the Hamiltonian $H_{N}^{C}+\lambda A_{N}^{C}$ (with $\delta=1$ ). It is easy to see that $f^{C}(\lambda)$ is concave in $\lambda$ 
and hence $\lim _{\lambda \downarrow 0} \lambda^{-1}\left[f^{C}(\lambda)-f^{C}(0)\right] \equiv G^{+}$and $\lim _{\lambda \downarrow 0} \lambda^{-1}\left[f^{C}(0)-f^{C}(-\lambda)\right]$ $\equiv G^{-}$exist everywhere. If $G^{+}=G^{-}$(i.e. the right derivative equals the left derivative) then by a theorem of Griffiths (6)

$$
\lim _{N \rightarrow \infty} \frac{d}{d \lambda} f_{N}^{C}(\lambda)=\frac{d}{d \lambda} f^{c}(\lambda)
$$

This is the case in which the classical expectation value $N^{-1}\left\langle A_{N}\right\rangle^{C}$ has a well defined limit. Call it $\alpha$. Then

$$
\lim _{J \rightarrow \infty} \lim _{N \rightarrow \infty} N^{-1}\left\langle A_{N}\right\rangle^{Q}=\alpha,
$$

as one sees by taking the limit $\lambda \rightarrow 0$ in (6.6). In other words, we have proved that for intensive observables, as defined above, the quantum expectation value equals the classical expectation value after first taking the thermodynamic limit and then taking the classical limit $J \rightarrow \infty$. If one takes the limits in the opposite order the theorem is trivially true and uninteresting. Note that we have not proved that the quantum thermodynamic limit, $\lim _{N \rightarrow \infty} N^{-1}\left\langle A_{N}\right\rangle^{Q}$ exists. It may not.

The same proof obviously goes through for ground state expectation values, as in Section VI.A, because the ground state energy is also concave in $\lambda$.

Acknowledgements. The author thanks the Institut des Hautes Etudes Scientifiques for its hospitality, as well as the Chemistry Laboratory III, University of Copenhagen where part of this work was done. The financial assistance of the Guggenheim Memorial Foundation is gratefully acknowleged. The author also acknowledges his gratitude to Dr. N. W. Dalton who suggested the problem to him in 1967.

\section{Appendix A: Some Useful Formulas}

The algebra $\mathscr{M}^{2 J+1}$ has $S_{+}, S_{-}$and $S_{z}$ as generators. Hence, the following generating function permits, by differentiation, easy calculation of $g(\Omega)$ in $(2.15)$ or Table 1 for any operator. It is to be found, with appropriate modifications, in Ref. [2].

$$
\begin{aligned}
& \left\langle\Omega\left|\exp \left(\gamma S_{+}\right) \exp \left(\beta S_{z}\right) \exp \left(\alpha S_{-}\right)\right| \Omega\right\rangle \\
& =\left\{\left[e^{-i \varphi} \sin \frac{1}{2} \theta+\gamma \cos \frac{1}{2} \theta\right]\left[e^{i \varphi} \sin \frac{1}{2} \theta+\alpha \cos \frac{1}{2} \theta\right] e^{-\beta / 2}+e^{\beta / 2}\left[\cos \frac{1}{2} \theta\right]^{2}\right\}^{2 J} .
\end{aligned}
$$

Turning to (2.13), we calculate $A_{G}$ for a sufficiently large class of functions $G(\Omega)$. Let

$$
G(\Omega)=e^{i m \varphi}\left(\cos \frac{1}{2} \theta\right)^{p}\left(\sin \frac{1}{2} \theta\right)^{q}
$$


where $m$ is an integer and $p$ and $q$ are complex numbers. Defining $A(m, p, q) \equiv A_{G}$, the matrix elements of this operator can be calculated using (2.7) to be

$$
\begin{aligned}
& A\left(m, p, q ; M, M^{\prime}\right)=\delta\left(M-M^{\prime}-m\right) \Gamma(J+\alpha+1+p / 2) \Gamma(J-\alpha+1+q / 2) \\
& \cdot[(J+\alpha+m / 2) !(J+\alpha-m / 2) !(J-\alpha-m / 2) !(J-\alpha+m / 2) !]^{-1 / 2} \quad(\text { A. } 3) \\
& \cdot(2 J+1) ! / \Gamma(2 J+2+p / 2+q / 2),
\end{aligned}
$$

where $\delta$ is the Kroenecker delta function, $\Gamma$ is the gamma function and $\alpha=\left(M+M^{\prime}\right) / 2$. This formula has been used to calculate Table 1 .

\section{Appendix B: Application to the One Dimensional Heisenberg Chain}

To illustrate the methods of this paper, we derive bounds for the free energy of a Heisenberg chain whose Hamiltonian is

$$
H=-\sum_{i=1}^{N-1} S^{i} \cdot S^{i+1} .
$$

Each spin is assumed to have angular momentum $J$. We have chosen the isotropic case for simplicity, but one could equally well handle the anisotropic Hamiltonian with a magnetic field. Note that $\beta>0$ is the ferromagnetic case while $\beta<0$ is the antiferromagnetic case.

The classical partition function is

$$
Z_{N}^{C}(\beta, x)=(4 \pi)^{-N} \int d \Omega_{N} \exp \left\{\beta x^{2} \sum_{i=1}^{N-1} \boldsymbol{\Omega}^{i} \cdot \boldsymbol{\Omega}^{i+1}\right\}
$$

with free energy per spin

$$
f^{C}(\beta, x)=-\lim _{N \rightarrow \infty}(N|\beta|)^{-1} \ln Z_{N}^{C}(\beta, x) .
$$

Our bounds are that

$$
f^{C}(\beta, J) \geqq f^{\mathcal{Q}}(\beta, J) \geqq f^{C}(\beta, J+1) .
$$

It is easy to evaluate (B.2) by the transfer matrix method. The normalized eigenfunction ( of $\Omega$ ) giving the largest eigenvalue is obviously the constant function $(4 \pi)^{-1 / 2}$. Thus,

$$
f^{c}(\beta, x)=-|\beta|^{-1} \ln A(\beta, x),
$$


where

$$
\begin{aligned}
A(\beta, x) & =(4 \pi)^{-1} \int d \Omega \exp \left\{\beta x^{2} \boldsymbol{\Omega} \cdot \boldsymbol{\Omega}^{\prime}\right\} \\
& =\left(\beta x^{2}\right)^{-1} \sinh \left(\beta x^{2}\right),
\end{aligned}
$$

and $A(\beta, x)$ is independent of $\Omega^{\prime}$ as it should be. In this approximation, (B.4), one cannot distinguish between the ferro- and antiferromagnetic cases as far as the free energy is concerned.

To illustrate the idea mentioned at the ends of Sections III and IV, we suppose that the chain has $2 N+1$ spins and we let $\mathscr{H}_{N}$ (resp. $\mathscr{H}$ ) be the Hilbert space for the odd (resp. even) numbered spins. $\mathscr{H}^{\prime}=\mathscr{H} \otimes \mathscr{H}_{N}$ is the whole space. Our bounds are

$$
g(\beta, J) \geqq f^{Q}(\beta, J) \geqq g(\beta, J+1),
$$

where

$$
\begin{aligned}
g(\beta, x) & =-\lim _{N \rightarrow \infty}(2 N|\beta|)^{-1} \ln \left\{(2 J+1)^{-N} \tilde{Z}_{N}(\beta, x)\right\} \\
\tilde{Z}_{N}(\beta, x) & =(4 \pi)^{-N} \int d \Omega_{N} \operatorname{Tr} \exp \left\{\beta x \sum_{i=1}^{N} \boldsymbol{S}^{2 i} \cdot\left(\boldsymbol{\Omega}^{2 i-1}+\boldsymbol{\Omega}^{2 i+1}\right)\right\}
\end{aligned}
$$

and where $d \Omega_{N}=d \Omega^{1} d \Omega^{3} \ldots d \Omega^{2 N+1}$ and the trace is over the Hilbert space of $S^{2}, S^{4}, \ldots, S^{2 N}$.

Since the remaining spin operators no longer interact, it is easy to calculate the trace. For a single spin:

$$
\operatorname{Tr} \exp [b \boldsymbol{S} \cdot \boldsymbol{v}]=\sum_{M=-J}^{J} \exp [b M v]
$$

where $b$ is a constant and $\boldsymbol{v}$ is a vector of length $v$. Now we can do the integration over $\mathscr{S}_{N}$ by the transfer matrix method (with the same eigenvector $(4 \pi)^{-1 / 2}$ ) and obtain

$$
g(\beta, x)=-\frac{1}{2}|\beta|^{-1} \ln [A(\beta, x) /(2 J+1)]
$$

where

$$
\begin{aligned}
A(\beta, x) & =(4 \pi)^{-1} \int d \Omega \sum_{M=-J}^{J} \exp \left\{\beta x M\left|\boldsymbol{\Omega}+\boldsymbol{\Omega}^{\prime}\right|\right\} \\
& =2 \int_{0}^{1} \mathrm{y} d y \sinh [(2 J+1) \beta x y] / \sinh [\beta x y] .
\end{aligned}
$$

Again, no distinction between the ferro- and antiferromagnetic cases appears. 


\section{References}

1. Millard, K., Leff, H.: J. Math. Phys. 12, 1000-1005 (1971).

2. Arecchi,F.T., Courtens, E., Gilmore, R., Thomas, H.: Phys. Rev. A6, 2211-2237 (1972).

3. Radcliffe, J. M.: J. Phys. A 4, 313-323 (1971).

4. Kutzner, J.: Phys. Lett. A 41, 475-476 (1972).

Atkins, P. W., Dobson, J.C.: Proc. Roy. Soc. (London) A, A 321, 321-340 (1971).

5. Golden, S.: Phys. Rev. B 137, 1127-1128 (1965).

6. Griffiths, R. B.: J. Math. Phys. 5, 1215-1222 (1964).

7. Hepp, K., Lieb, E.H.: The equilibrium statistical mechanics of matter interacting with the quantized radiation field. Preprint.

E. H. Lieb

I.H.E.S.

F-91440 Bures-sur-Yvette, France 